\title{
EXPERIMENTAL INVESTIGATIONS ON THE INFLUENCE OF PRESTRESS ON LAMB WAVE PROPAGATION
}

\author{
$\underline{\text { T. Barth }}^{1 *}$, R. Lammering ${ }^{1}$ \\ ${ }^{1}$ Helmut-Schmidt-University / University of the Federal Armed Forces - Institute of Mechanics, \\ Holstenhofweg 85, 22043 Hamburg, Germany \\ *bartht@hsu-hh.de
}

The use of Lamb waves in structural health monitoring is a well-known approach in today's mechanical engineering [2,4]. Although the behavior of Lamb waves is widely explored, one of the topics that has not been covered in all its depths is the influence of prestress on the behavior of Lamb waves. One of the challenges concerning this topic is to obtain reliable measurement data which can be used to verify numerical simulations. There are a few experimental approaches to measure these influences, for example $[1,3]$. When considering these approaches, it is noticeable that the occurring influences are only investigated at a few single frequencies. Therefore the verification of numerical models over larger frequency ranges is not possible.

The aim of the approach shown here is to extend the experimental tests to large frequency ranges in order to obtain a better understanding of the influence prestress has on the propagation of Lamb waves. It should be additionally noted that in most frequency ranges this influence is very small, therefore a method has been developed which puts special emphasis on high accuracy and automatization. The measurements are performed by a Doppler laser vibrometer, which captures the out-of-plane velocities along a path on a prestressed specimen. For excitation, a piezo electric actuator is used, which generates Lamb waves in the specimen using multi-frequency signals. The data is analyzed using a combination of $2 \mathrm{~d}$ Fourier transform and an automated peak detection so that the frequencies and wave numbers that occur can be determined. To verify the method, measurements in unstressed specimens are compared with analytical solutions. In this case, a good agreement is obtained.

The received results are capable of giving a better understanding of the influences of prestress on Lamb waves and a help to give a correct interpretation of signals obtained from structural health monitoring systems. Further, they can be used for the assessment of numerical simulations. Although the results presented are limited to aluminum (AlMg3) for now, the methodology is material independent so that more complex material systems will be investigated in the future, for example fiber-metal laminates.

\section{References}

[1] Gandhi, N., Michaels, J., Lee, S.J.: Acoustoelastic lamb wave propagation in biaxially stressed plates. The Journal of the Acoustical Society of America 132, 1284 - 93 (2012)

[2] Giurgiutiu, V.: Structural Health Monitoring. Academic Press, Burlington (2008)

[3] Qiu, L., Yan, X., Lin, X., Yuan, S.: Multiphysics simulation method of lamb wave propagation with piezoelectric transducers under load condition. Chinese Journal of Aeronautics 32(5), 1071 - 1086 (2019)

[4] Rose, J.L.: Ultrasonic Guided Waves in Solid Media. Cambridge University Press (2014) 